\title{
Generating (co)homological information using boundary scale
}

\author{
Helena Molina-Abril a ${ }^{a}$ Pedro Real ${ }^{a}$ * ${ }^{*}$ Fernando Díaz-del-Río ${ }^{b}$ \\ a Department of Applied Mathematics I. University of Seville Spain \\ ${ }^{\mathrm{b}}$ Department of Computer Architecture and Technology University of Seville Spain
}

Keywords:

Geometric cell complex

Algebraic-topological model

Scale-space model

Homology groups

Hierarchical graph

\begin{abstract}
A B S T R A C T
In this paper we develop a new computational technique called boundary scale-space theory. This technique is based on the topol ${ }^{1}$ ogical paradigm consisting of representing a geometric subdivided object $K$ using a one-parameter family of geometric objects $\left\{K_{i}\right\}_{i} \geq 1$ all of them having the same number of closed pieces than $K$. Each piece of $K_{i}(\forall i \geq 1)$ presents the same interior part than the corresponding one in $K$, and a different boundary part depending on the scale $i$. Working with coefficients in a field, a scale is installed for the algebraic boundary of each piece and a new invariant for cell complex isomorphisms is given in terms of the Betti numbers of the generated boundary-scalespace cell complexes. Moreover, the so called homology boundary scale-space model of $K$ ( $h b s s$-model for short) is introduced here. This

model consists of a hierarchical graph whose nodes are the homology generators of the different bound- ary scale levels and whose edges are specified by homology generators of consecutive boundary scale

indices linked by (hbss-transition maps) preserving homology classes. Various codes for each connected subgraph of an hbss -model are defined, which besides being fast and efficient similarity measures for cel- lular structures, they are as well relevant interpretive tools for the hbss -model. Finally, experimentations mainly aimed at clarifying and understanding the notion of $h b s s$-model, as well as conjecturing about new graph isomorphism invariants (seeing graphs as a 1-dimensional cell complexes), are performed.
\end{abstract}

\section{Introduction}

Topological exploration of complex data sets is a key issue in Data Science. In this direction, Persistent Homological Data Analysis is a successful set of techniques, that uses the topological feature of persistent homology as its main analysis tool (see [1,2]). The inference problem of defining and computing the homology of a cloud dataset is solved by constructing a cell complex structure (see, for instance, [3] for a review of this notion). Persistence homology is mainly based on obtaining homological information, that depends on a filtration of such initial cell complex. The essential idea to use persistent homology is to generate a viewpointdependent representation of high-dimensional data sets, in which the homology classes that persist the longest through this filtration are highlighted.

In this paper, we are interested in searching analytical tools based on a different topological paradigm. Geometric cell complexes can be seen here as structures constituted by closed cells in which their corresponding interior (connectivity piece) and boundary frames (connectivity capacity of the piece) can be separately

\footnotetext{
* Corresponding author.

E-mail address: real@us.es (P. Real).

1 comples -> complex
}

manipulated. In the classical scale-space theory (see [4] for a review), "the quintessence is that scale provides topology" (see [5]). In the proposed boundary scale-space model (bss-model, for short), "topology provides scale". Given a geometric cell complex $K$, the connectivity pieces for each level of the bss-model are always the interior parts of the different dimensional cells of $K$. On the other hand, the connectivity capacity of each piece at a certain boundary scale level changes, following a common structural boundary pattern. Let us emphasize that this idea is different from the classical morphological scale-space paradigm in image analysis, in which the change of scale affects both, the set of connectivity pieces and the capacities of the cells (see [6,7]).

The proposed bss-model handles a given cell complex $K$ at different boundary scales, by representing $K$ as a one-parameter family $\left\{K_{i}\right\}_{i \geq 1}$ of cell complexes, having all of them the same number of closed cells than $K$, with identical interior parts than $K$, but different boundaries that are "measured" in terms of a scale parameter. The boundary scale parameter $i$ is a positive integer specifying the common "topological size" of the boundary of the cells of the same dimension at that level.

In Fig. 1, a set of local geometric boundary operators of a 2dimensional square that are susceptible to be applied in a bssmodel are shown withing a square regular cell complex of size $7 \times 7$. 

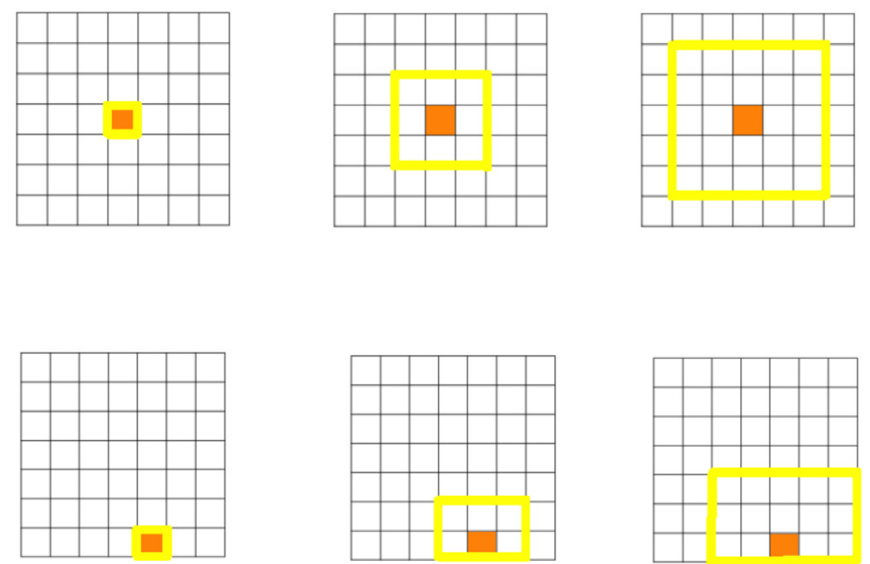

Fig. 1. Different boundaries (in yellow) of a 2-dimensional cell (interior part in orange) within a regular square cell complex of size $7 \times 7$.

In order to develop a computationally viable method, we place the bss-theory in the context of an algebraic scenario and use homology features for determining a homology boundary scale-space model (hbss-model, for short) for a geometric cell complex $(K, \partial)$. Concretely, working with coefficients in a field $\mathbb{F}$, the algebraic bssmodel of the canonically associated chain complex $\left(\mathbb{F}_{*}[K], \partial\right)$ (being $\mathbb{F}_{*}[K]$ the vector space of finite linear combinations of cells of $K)$ is a one-parameter family $\left\{\left(\mathbb{F}[K], \partial^{i}\right)\right\}_{i \geq 0}$ of chain complexes satisfying that $\partial^{1}=\partial, \partial \partial^{i}=0=\partial^{i} \partial(\forall i \geq 1)$ and $\partial^{i} \partial^{j}=0(\forall i, j \geq 1)$. With these conditions, we guarantee that all the homology boundary chain complexes have the same Euler-Poincare characteristic than $K$ through all the levels of the hbss-model. The one-parameter family $\left\{\beta\left(\mathbb{F}[K], \partial^{i}\right)\right\}_{i \geq 0}$ of vectors $\beta\left(\mathbb{F}[K], \partial^{i}\right)=\left(\beta_{0}^{i}, \ldots, \beta_{0}^{n}\right)$ formed by the respective Betti numbers of $\left(\mathbb{F}[K], \partial^{i}\right.$ ) (given in increasing dimension order from left to right) turns out to be an invariant for cell complex isomorphisms.

The hbss-model consists of a hierarchical graph whose nodes are homology generators of different boundary scale levels and whose edges are specified by homology generators of consecutive boundary scale indices linked by homology-maps ( $h b s s$-transition maps). All this information can be straightforwardly extracted from a family of algebraic-topological models of $\left(\mathbb{F}[K], \partial^{i}\right), i \geq 1$ (ATmodels, for short, see [8-11]).

Intuitively, the goal of hbss-models is to keep track of all the homological information of a given cell complex that is dependant of the boundary scale. Let us note that the hbss-model does not satisfy in general the causality principle (see [12]), that states that for any pair of scales $i$ and $j$ with $i<j$, homological information at scale $j$ finds a "cause" at scale $i$. This principle is satisfied when it is restricted to a connected subgraph of the model. In order to clarify, understand and value the topological discriminatory power of the hbss-model, we work with lossy codes for each connected subgraph of an hbss-model.

The following subsection of the paper summarizes some of the published works that are related to the proposed model. Section 2 is devoted to recall the combinatorial and algebraic machinery for computing the hbss-model. Next, the construction of this models is presented in Section 3. Section 4 shows some examples that have been computed using an ad hoc implementation of the method. Finally, the paper concludes in Section 5.

\subsection{Related works}

Concerning homotopy representation models of digital objects and images, there are numerous works arising from digital topology sources [13-15], continuous or cellular topology [16-18] and
$\mathrm{nD}$ shape search in which three clearly differentiated concepts appear: Reeb graphs [19,20], skeletons [21-23] and boundary representations [24-26]. Relative to the homology of cell complexes there is plenty of literature, mainly based on a pure algebraic perspective, that is devoted to its computation. The classical method is based on the diagonalization of cell-incidence matrices to Smith normal form (SNF) (see [27]). In the following decades, some advances in the computation of the SNF have been achieved (see [28]), but the most successful approaches consist of reducing the number of cells in the complex using discrete-vector-field dynamics (Discrete Morse theory [29]) before computing the SNF for the small resulting cell complex (see, for instance, [30-35]). In this paper we go beyond homological computation and design an algorithm for computing a new representation based on homology. In this sense, boundary scale-space theory extends and improves both the algebraic model called Algebraic-Topological model (see [8-11]) and the combinatorial model called Homological Spanning Forest (HSF, for short, see $[32,36,37]$ ), with respect to their aim of developing new topological representations within the digital object's context.

\section{Preliminaries}

We work in this paper with cell complex representations (composed of cells and bounding relations between them), that allow to model, for example, not only an $n$-dimensional digital object at sub- $n$-xel level but also significant algebraic (co)homological information (with coefficient in a field $\mathbb{F}$ ).

First of all, we provide a slightly modified version of the classical abstract cell complex notion (see [3] for a survey).

We say that $\mathrm{K}=(K, B, d m s)$ is an abstract cell complex (or $A C C$, for short) if:

- $C=\left\{K_{q}\right\}_{q \in \mathbb{N} \cup\{0\}}$ is a finite set with a gradation dms :K $\rightarrow \mathbb{N} \cup\{0\}$ defined by $\operatorname{dms}(c)=q$ for $c \in K_{q}$;

- $B: K \times K \rightarrow \mathbb{N} \cup\{0\}$ is a map such that satisfies the following condition: $B\left(c, c^{\prime}\right) \neq 0$ implies $c \in K_{q-1}, c^{\prime} \in K_{q}$.

We refer to the elements of $C$ as cells and to $B\left(c, c^{\prime}\right)$ as the bounding function of the ACC K applied to the couple $\left(c, c^{\prime}\right)$. If we extend the bounding function of the ACC in an antisymmetric and transitively way, we recover the classical notion of ACC.

The connectivity-graph $G(K, B, d m s)=(V, E)$ of an abstract cell complex $(K, B, d m s)$ is the graph whose set of nodes is $K$ and an edge $\left\{c, c^{\prime}\right\} \in E$ if $B\left(c, c^{\prime}\right)$ or $B\left(c^{\prime}, c\right)$ is different from zero.

Now, let us define the (algebraic) notion of geometric cell complex. We say that $\mathrm{K}=(K, \kappa, d m s)$ is a Lefschetz complex ([38]) if:

- $K=\left\{K_{q}\right\}_{q \in \mathbb{N} \cup\{0\}}$ is a finite set with a gradation dms: $K \rightarrow$ $\mathbb{N} \cup\{0\}$ defined by $\operatorname{dms}(c)=q$ for $c \in K_{q}$;

- $\kappa: K \times K \rightarrow \mathbb{F}$ is a map such that $\kappa\left(c, c^{\prime}\right) \neq 0$ implies $c \in K_{q-1}$, $c^{\prime} \in K_{q}$. For any $c, c^{\prime \prime} \in K$ we have $\sum_{c^{\prime} \in K} \kappa\left(c, c^{\prime}\right) \kappa\left(c^{\prime}, c^{\prime \prime}\right)=0$.

We refer to the elements of $C$ as cells and to $\kappa(x, y)$ as the incidence coefficient of $x, y$.

We are interested here in Lefschetz complexes satisfying that for any $c, c^{\prime} \in C$ the incidence coefficient $\kappa\left(c, c^{\prime}\right)$ is either zero or \pm 1 of $\mathbb{F}$. These structures are simply called here geometric cell complexes. The identity function $1_{K}: K \rightarrow K$ is defined by $1_{K}(c)=c, \forall c \in K$. Associated to a geometric cell complex $(K, \kappa$, $d m s)$, there is a bounding function $B: K \times K \rightarrow \mathbb{N} \cup\{0\}$ defined by $B\left(c, c^{\prime}\right)=1$ if $\kappa\left(c, c^{\prime}\right) \neq 0$ and $B\left(c, c^{\prime}\right)=0$ otherwise. If $c \in K$ and $R$ is a set of cells of $(K, \kappa, d m s)$, we define the bounding function $B(c, R)=\sum_{c^{\prime} \in R} B\left(c, c^{\prime}\right)\left(\right.$ resp. $\left.B(R, c)=\sum_{c^{\prime} \in R} B\left(c, c^{\prime}\right)\right)$.

In a purely algebraic context, a geometric cell complex $(K, \kappa$, $d m s)$ is determined by a free chain complex $(\mathbb{F}[K], \partial)$ with differential or algebraic boundary $\partial_{q+1}: \mathbb{F}[K]_{q+1} \rightarrow \mathbb{F}[K]_{q}(q=0,1,2, \ldots)$ defined on generators (linear map) by $\partial_{q+1}(c)=\sum_{c^{\prime} \in K} \kappa\left(c^{\prime}, c\right) c^{\prime}$. A 
chain map $f_{q}: \mathbb{F}\left[K_{q}\right] \rightarrow \mathbb{F}\left[K_{q}^{\prime}\right]$ is a linear map satisfying $f_{q-1} \partial_{q}=$ $\partial_{q}^{\prime} f_{q}(q \geq 0)$. Its codifferencial or algebraic coboundary $\delta_{q}$ : $\mathbb{F}\left[K_{q}\right] \rightarrow \mathbb{F}\left[K_{q+1}\right](q \geq 0)$ is defined on generators by $\delta_{q}(c)=$ $\sum_{c^{\prime} \in K} \kappa\left(c, c^{\prime}\right) c^{\prime}$. Its homology (resp. cohomology) groups $\left\{H_{q}(K, \kappa\right.$, $d m s)\}$ (resp. $\left.\left\{H^{q}(K, \kappa, d m s)\right\}\right)$ are defined as the homology groups $\left\{H_{q}([F](K), \partial)\right\}$ of the chain complex $(\mathbb{F}[K], \partial)$ (resp. of $(\mathbb{F}[K], \delta)$ ).

Hence, $H_{q}(K, \kappa, d m s)=\operatorname{Ker}\left(\partial_{q}\right) / \partial_{q+1}(K)\left(\operatorname{resp} . H^{q}(K, \kappa, d m s)=\right.$ Ker $\left.\left(\delta_{q+1}\right) / \delta_{q}(K)\right)$. The $n^{\text {th }}$ Betti number represents the rank of the $n^{\text {th }}$ homology group.

Let us note that if $f: \mathbb{F}[K] \rightarrow \mathbb{F}[K]$ is a linear map, Ker $f=\{c \in$ $\mathbb{F}[K] / f(c)=0\}$ and $f(C)=\left\{c^{\prime} \in \mathbb{F}[K] / c^{\prime}=f(c)\right\}$.

The definitions of homology make sense due to the fact that $\partial_{q+1} \partial_{q}=0$ and $\delta_{q} \delta_{q+1}=0, \forall(q \geq 0)$.

From now on, we use the hybrid combinatorial-algebraic notation $(K, \partial)$ for describing a geometric cell complex. The notation $c^{\prime} \in f$, being $f \in \mathbb{F}[K]$ indicates that the cell $c^{\prime}$ is involved as a nonnull addend of this linear combination.

It is straightforward to specify geometric cubical cell complexes modeling $n$-dimensional digital images at sub- $n$-xel level. In fact, we are mainly interested in testing the designed algorithms in digital objects (that are previously cellularized or polyhedrized), in order to progress in topological acuity and representation within the digital image context.

\subsection{AT-models}

Taking into consideration the previous definitions, we are now able to define an AT-model $(K, \partial, \phi)$ of a geometric cell complex $(K, \partial)$. The homomorphism $\phi_{q}: \mathbb{F}\left[K_{q}\right] \rightarrow \mathbb{F}\left[K_{q+1}\right]$, called integral operator, satisfies the following three conditions: (a) $\phi \phi=0$; (b) $\partial \phi \partial=\partial$; (c) $\phi \partial \phi=\phi$.

Let us emphasize that the integral operator is, in particular, a codifferential operator.

An explicit homology equivalence between the chain complex $(\mathbb{F}[K], \partial)$ and a free chain complex with null differential (which, obviously, is isomorphic to the homology $H(K, \partial)$ ) can be constructed using AT-models. The germ idea of the AT-model theory comes back to the original notion of chain contraction, used by Samuel Eilenberg and Saunder Mac Lane in their works of homological computation (see, for example, [39]). If $(K, \partial)$ and $\left(K^{\prime}\right.$, $\left.\partial^{\prime}\right)$ are two geometric cell complexes (in fact, we take the associated chain complexes), a chain contraction is a triple $(f, g, \psi)$ of chain maps $f_{q}: \mathbb{F}\left[K_{q}\right] \rightarrow \mathbb{F}\left[K_{q}^{\prime}\right], g_{q}: \mathbb{F}\left[K_{q}^{\prime}\right] \rightarrow \mathbb{F}\left[K_{q}\right]$ and $\psi_{q}: \mathbb{F}\left[K_{q}\right] \rightarrow$ $\mathbb{F}\left[K_{q+1}^{\prime}\right]$ satisfying the following conditions: (a) $1_{\mathbb{F}[K]}=g f+\partial \psi+$ $\psi \partial$; (b) $f g=1_{\mathbb{F}\left[K^{\prime}\right]}$; (c) $f \psi=0$; (d) $\phi g=0$; (e) $\psi \psi=0$. For instance, a chain contraction $(f, g, \psi)$ that can be specified from an AT-model $(K, \partial, \phi)$ has $\psi=\phi, f=1_{\mathbb{F}[K]}-\phi \partial-\partial \phi$ and $g$ : $\operatorname{Im}\left(1_{\mathbb{F}[\mathrm{K}]}-\phi \partial-\partial \phi\right) \rightarrow \mathbb{F}[\mathrm{K}]$ (inclusion map).

The pseudocode for computing an AT-model (in fact, the integral operator defining the AT-model) of a finite geometric cell complex $\mathrm{K}$ is shown in Algorithm 1. The Betti numbers of $\mathrm{K}$ can be straightforwardly extracted form the final value of the $\mathcal{J}$ variable in the algorithm (line 24), by counting the number of critical cells of each dimension.

\subsection{Homology-preserving maps}

Another fundamental piece of the boundary scale-space theory are homology-preserving maps:

Definition Given two chain complexes $\left(C_{*}, d\right)$ and $\left(C_{*}^{\prime}, d^{\prime}\right)$, a linear map $f:\left(C_{*}, d\right) \rightarrow\left(C_{*}^{\prime}, d^{\prime}\right)$ is a pseudo-chain map or homologypreserving map if:

- $f(\operatorname{Ker} d) \subset \operatorname{Ker} d$;

- $f\left(d\left(C_{*}\right) \subset d\left(C_{*}\right)\right.$.
Algorithm 1 [AT-model construction]

Input:A geometric cell complex $\mathrm{K}:=\{K, \partial, d m s\} . C$ a list with all the cells of $\mathrm{K}$ ordered by increasing dimension $c_{1}^{0}, \ldots c_{\ell_{1}}^{0}, c_{1}^{1}, \ldots$, $c_{\ell_{2}}^{1}, \ldots, c_{1}^{n}, \ldots, c_{\ell_{n}}^{n}$.Here, $\operatorname{dms}\left(c_{j}^{k}\right)=k, \forall k, j$ and $\sum_{1 \leq q \leq n} \ell_{q}=\ell$. Let us also use the cell ordering $c_{j}^{k}=c_{j+\sum_{q<k} \ell_{q}}$.The boundary operator $\left.\partial\right|_{\mathbb{F}\left[c_{1}, \ldots, c_{i}\right]}$ is denoted by $\partial_{i}$.

1: $\mathcal{H}_{0}^{\partial} \leftarrow \emptyset ; \mathcal{L}_{0}^{\phi} \leftarrow \emptyset ; \mathcal{J}_{0}^{\phi} \leftarrow \emptyset ; \mathcal{S}_{0}^{p r} \leftarrow \emptyset ;$

2: for $k=0$ to $n$ do

3: $\quad$ for $j=1$ to $\ell_{k}$ do

4: $\quad i \leftarrow j+\sum_{q<k} \ell_{q}$;

5: $\quad \phi_{i-1}\left(c_{i}\right) \leftarrow 0$;

6: $\quad B n d_{i} \leftarrow\left\{e \in \partial_{i}\left(c_{i}\right)\right\} ; \quad \triangleright$ Boundary of the current cell

7: $\quad \bar{c}_{i} \leftarrow c_{i}+\phi_{i-1} \partial_{i}\left(c_{i}\right) ; \quad \triangleright$ Potential cycle assoc. to $c_{i}$

8: $\quad \overline{B n d}_{i} \leftarrow\left\{e \in \partial_{i}\left(\bar{c}_{i}\right)\right\} ; \quad \triangleright$ Algebraic boundary of $\bar{c}_{i}$

9: $\quad \mathcal{H}_{i}^{\partial} \leftarrow H_{i-1}^{\partial} \bigcup\left\{\bar{c}_{i}\right\} ; \mathcal{L}_{i}^{\phi} \leftarrow \mathcal{L}_{i-1}^{\phi} \bigcup\left\{c_{i}\right\} ;$

10: $\quad \mathcal{J}_{i}^{\phi} \leftarrow \mathcal{J}_{i-1}^{\phi} \cup\left\{c_{i}\right\} ; \quad \triangleright$ Homology generators, critical cells

$\begin{array}{ccc}\text { 11: } & \text { if } \partial_{i}\left(\bar{c}_{i}\right)==0 \text { then } \\ \text { 12: } & \text { for } r=1 \text { to } i \text { do } \\ \text { 13: } & \phi_{i}\left(c_{r}\right) \leftarrow \phi_{i-1}\left(c_{r}\right) ; \\ \text { 14: } & \text { else } & \triangleright \text { In case } c_{i} \text { does not generate a cycle }\end{array}$

15: $\quad$ Choose one of the cells $\mathbf{e} \in \overline{\mathrm{Bnd}}_{i}$;

16: $\quad$ Choose one of the cells $\overline{\mathbf{e}} \in \mathrm{Bnd}_{i}$

17: $\quad \tilde{\phi}(\mathbf{e}) \leftarrow c_{i}$;

18: $\quad \tilde{\phi}(c) \leftarrow 0$ for each $c \in \mathcal{L}_{i-1}^{\phi} \backslash\{\mathbf{e}\}$;

19: $\quad \overline{\mathbf{e}} \leftarrow \mathbf{e}+\phi_{i-1} \partial_{i}(\mathbf{e})$;

20: $\quad$ for $q=1$ to $i-1$ do

21: $\quad \phi_{i}\left(c_{q}\right) \leftarrow\left(\phi_{i-1}+\left(i d_{\mathrm{C}_{i}}-\phi_{i-1} \partial_{i-1}\right) \tilde{\phi}\left(i d_{\mathrm{C}_{i}}-\right.\right.$ $\left.\left.\partial_{i-1} \phi_{i-1}\right)\right)\left(c_{q}\right), \forall c_{q} \in C_{i}$

22: $\mathcal{H}_{i}^{\partial} \leftarrow \mathcal{H}_{i}^{\partial} \backslash\left\{\overline{\mathbf{e}}, \bar{c}_{i}\right\} ; \quad \quad$ Updating homology generators

23: $\quad \mathcal{L}_{i}^{\phi} \leftarrow \mathcal{L}_{i}^{\phi} \backslash\{\mathbf{e}\} ; \quad \triangleright$ Updating comb. homology kernel

24: cells

$$
\mathcal{J}_{i}^{\phi} \leftarrow \mathcal{J}_{i}^{\phi} \backslash\left\{\mathbf{e}, c_{i}\right\} ; \quad \triangleright \text { Updating set of critical }
$$

\section{Output:}

- An AT-model $\left(C, \partial_{\ell}, \phi_{\ell}, d m s\right)$ and a combinatorial basis (set of critical cells) specified by $\mathcal{J}_{\ell}^{\phi}$ and ordered by increasing dimension $\left\{c r t_{1}^{d_{1}}, \ldots, c r t_{m}^{d_{m}}\right\}$ (with $\operatorname{dms}\left(\operatorname{crt}_{q}^{d_{q}}\right)=$ $\left.d_{q}, 1 \leq q \leq m\right)$ of the homology $H(C, \phi)$. A set of representative cycles of the homology classes with regards to $\partial$ is given by $\mathcal{H}_{\ell}^{\phi}$.

In other words, the map $[f]_{d}: H\left(C_{*}, d\right) \rightarrow H\left(C_{*}^{\prime} d^{\prime}\right)$ passing the map $f$ to homology is well-defined. A chain map is also called a strong homology-preserving map. A homology-preserving map which is not a chain map is called a weak homology-preserving map

In particular, the equality $d^{\prime} f d=0$ is satisfied for homologypreserving maps.

To our knowledge, the first reference dealing with homologypreserving maps appears in the work of Levine in [40].

Examples of weak homology-preserving maps are the following:

- Let $\left(C_{*}, \partial, \phi\right)$ be an AT-model of a geometric cell complex. Let us consider the map $h(\phi, \partial)=1_{C}+\phi \partial$. On one hand, $h(\phi, \partial) \partial=\partial+\phi \partial \partial=\partial$. On the other, $\partial h(\phi, \partial)=\partial+\partial \phi \partial=$ $(1+\partial \phi) \partial=0$. It is easy to prove that $h(\phi, \partial)$ is a weak 
homology-preserving map. Analogously, we can prove that $h(\partial, \phi)=1_{C_{*}}+\partial \phi:\left(C_{*}, \partial\right) \rightarrow\left(C_{*}, \partial\right)$ is also a weak homologypreserving map.

- If $(C, \partial)$ is a geometric cell complex, then the composition $\partial \delta$ : $(C, \partial \delta \partial) \rightarrow(C, \partial)$ is a weak homology-preserving map.

Finally, we need a convenient definition of homologypreserving equivalence between two chain complexes.

Definition 2.1. Given two chain complexes $\left(C, d_{C}\right)$ and $\left(C^{\prime}, d_{C^{\prime}}\right)$, a homology-preserving equivalence (hpe, for short) between them is a pair of maps $(f, g)$, such that:

- $f:\left(C, d_{C}\right) \rightarrow\left(C^{\prime}, d_{C^{\prime}}\right)$ and $g:\left(C^{\prime}, d_{C^{\prime}}\right) \rightarrow\left(C, d_{C}\right)$ are two homology-preserving maps;

- $[g]_{d^{\prime}}[f]_{d}$ is the identity $1_{H(C)}: H_{*}\left(C, d_{C}\right) \rightarrow H_{*}\left(C, d_{C}\right)$ at homology level.

It is straightforward to prove that given an hpe from $\left(C, d_{C}\right)$ to $\left(C^{\prime}, d_{C^{\prime}}\right)$, their respective Euler-Poincaré characteristics are the same. Any chain contraction is in particular an hpe. In general, an hpe does not preserve Betti numbers.

A simple example of hpe is provided by the following proposition:

Proposition 2.1. Let $\left(C_{*}, d, \phi\right)$ be an AT-model of a finite chain complex $\left(C_{*}, d\right)$. The pair of chain maps $(h(d, \phi), h(\phi, d))$ constitutes an hpe from $\left(C_{*}, d\right)$ to $\left(C_{*}, 0\right)$.

This means, that the Euler-Poincaré characteristic $\chi\left(C_{*}, \partial\right)$ is equal to $\chi\left(C_{*}, 0\right)$. Therefore, we deduce a well-known result of computational topology: the Euler-Poincaré characteristic of $\left(C_{*}, \partial\right)$ in terms of Betti numbers agrees with the alternate sum of the cardinals of the sets of cells of different dimension.

\section{The boundary scale-space model}

Given a linear map $\psi:(C, d) \rightarrow\left(C^{\prime}, d^{\prime}\right)$ between chain complexes, the notation $\psi^{(n)}$ means the composition $\psi \ldots \psi n \in \mathbb{N}$.

Given a finite geometric cell complex $(K, \partial)$, it is straightforward to prove that any operator of the kind $\partial(\delta \partial)^{(m)}\left(\operatorname{resp} . \delta(\partial \delta)^{(m)}\right)$ $n \geq 0$ ) is again a differential (resp. a codifferential) of $K_{*}$. Let us note that $(\delta \partial)^{(0)}=(\partial \delta)^{(0)}=1_{\mathbb{F}[K]}$. Another type of differential (resp. codifferential) operators which can be derived from $\partial$ (and, consequently, $\delta$ ) are given by the formula $\partial \sum_{i=0}^{m}(\delta \partial)^{(i)}(m \geq 0)$.

In this paper, we will use $t(n)=\partial \sum_{i=0}^{n-1}(\delta \partial)^{i}(n \geq 1)$ to build the boundary scale model. Further analysis with other possibilities will be performed in the future. The differential $t(0)$ coincides with the zero map.

The idea of the boundary scale-space model is to analyze the algebraic information related to the finite sequence of the homology vector spaces $H(K, t(1)), H(K, t(2)), \ldots H(K, t(n))$.

For example, in the case of $(K, \partial)$ being a three-dimensional geometric complex, each homology $H(K, t(i))$ is a vector of three components $\left(H_{0}(K, t(i)), H_{1}(K, t(i)), H_{2}(K, t(i))\right)$, each one considered here as a set with as many elements as (in case of $H_{0}$ ) connected components, (in case of $H_{1}$ ) tunnels and (in case of $\mathrm{H}_{2}$ ) cavities the cell complex $K$ has. In order to know how this stratified information at consecutive boundary scales $i$ and $i+1$ is linked one to each other, we use the chain maps $h p e\left(d^{i}, d^{i+1}, \phi^{i}, \phi^{i+1}\right)$ of Proposition 2.1 applied to $(K, \partial)$, where $d^{i}=t(i), d^{i+1}=t(i+1)$ and $\phi^{i}$ and $\phi^{i+1}$ are the respective integral operators of AT-models $\left(K_{*}, t(i), \phi^{i}\right)$ and $\left(K_{*}, t(i+1), \phi^{i+1}\right)$. In fact, these maps guarantee that the homological transference in each transition of the boundary scale-space model is a homologically consistent process. They form the set of boundary scale-space transition maps between boundary scales $i$ and $i+1$.

We are now able to provide a definition of a boundary scalespace model for a geometric cell complex.

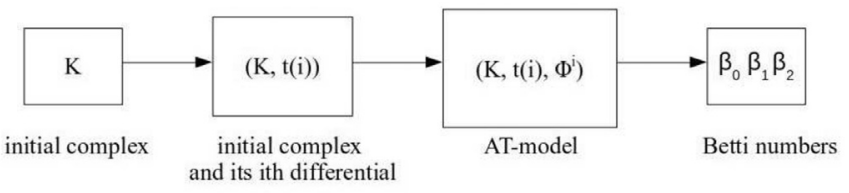

Fig. 2. Flowchart corresponding to the ith iteration of the hbss-model construction algorithm.

Definition 3.1. Let $(K, \partial)$ and $b s s=(t(1)=\partial, t(2), t(3), \ldots$.$) be a$ finite geometric cell complex and its boundary scale, respectively. We use the following ingredients in the construction of our particular $h b s s$-model $B S^{2}(K, \partial, b s s)$ :

- a sequence of AT-models $\left(K, t(i), \phi^{i}\right)(i \geq 1)$ and their associated chain contractions $\left(f^{i}, g^{i}, \phi^{i}\right)$ strongly connecting $(K, t(i))$ with its homology $H_{*}(K, t(i))$.

- a sequence of homology boundary scale-space transition maps $m_{i+1}^{i}: H(K, t(i+1)) \rightarrow H(K, t(i))$ (hbss transition maps, for short) given by:

$$
m_{i+1}^{i}=f^{i}\left(1+\phi^{i} t(i)\right)\left(1+t(i+1) \phi^{i+1}\right) g^{i+1}, \forall i \geq 1 .
$$

$B S^{2}(K, \partial, b s s)$ is then the hierarchical graph having as nodes at boundary scale $i$ the homology generators of $(K, t(i))$ and as edges the homology generators at consecutive levels $j$ and $j+1$ linked by the hbss transition map $m_{j+1}^{j}$.

This last definition makes it possible to establish a complete traceability of each homology generator at any boundary scale.

Starting from a homology preserving map $h:(K, \partial) \rightarrow\left(K^{\prime}, \partial\right)$ going from a geometric cell complex to another, it is also possible to straightforwardly define a family of maps $h_{i \geq 1}^{i}$ between their respective hbss-models, being each of them the appropriate composition of $h b s s$-transition maps for $(K, \partial)$ and $\left(K^{\prime}, \partial^{\prime}\right)$ and the map $h$.

\section{About the interpretability of the boundary scale-space model}

The construction of the hbss-model is given in polynomial time, due to the fact that the main expensive step is the generation of AT-models at different boundary scale levels. $O\left(n^{3}\right)$ elementary operations (being $n$, the number of cells of a geometric cell complex $(K, \partial)$ ) are needed for generating an AT-model of $(K, \partial)$. The flowchart showing each iteration of the algorithm is presented in Fig. 2.

The experimentation carried out in this section makes a headway on: (a) understanding, interpreting and analyzing the stability of the model (under homotopy-preserving changes) and (b) discriminative power of the model for distinguishing nonhomeomorphic 1-dimensional geometric object. An ad-hoc implementation of the hbss-model has been developed for this purpose and our tests will be executed with small instances of cell complexes.

Within $B S^{2}(K, \partial, b s s)$, it is now possible to tag with the same label homology generators (elements of a basis of the vector space $H(K, t(i)))$ that are connected via transition maps and, consequently, to specify hierarchical homological boundary scale-space connected graphs (hbss-graph, for short) of homology generators of the same dimension dms.

Fixing a maximal boundary scale index $m$ in our study of the boundary scale-space model, each hbss-graph of label $\ell$ and dimension $d m s$ is a hierarchical graph whose "birth" is at a certain initial boundary scale-space $k_{1}$, having nodes in the consecutive boundary scale levels until no more connected nodes are found at a certain level or "death". Each hbss-graph can admit multiple 


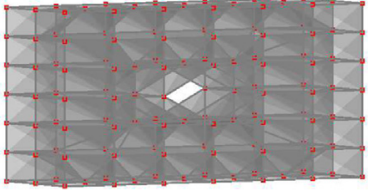

a)

Fig. 3. A cell complex of a torus and its corresponding hbss-Betti sequence for the first three levels.

\begin{tabular}{|c|c|c|c|}
\hline $\mathbf{i t}$ & $\boldsymbol{\beta} \mathbf{0}$ & $\boldsymbol{\beta} \mathbf{1}$ & $\boldsymbol{\beta 2}$ \\
\hline $\mathbf{1}$ & 1 & 2 & 1 \\
\hline $\mathbf{2}$ & 15 & 30 & 19 \\
\hline $\mathbf{3}$ & 15 & 62 & 75 \\
\hline $\boldsymbol{\ldots}$ & $\boldsymbol{\ldots}$ & $\boldsymbol{\ldots}$ & $\boldsymbol{\ldots}$ \\
\hline
\end{tabular}

b) a)

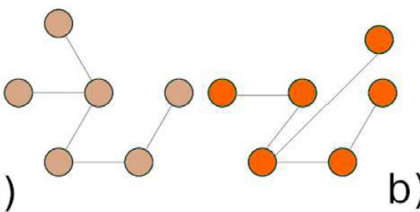

C)

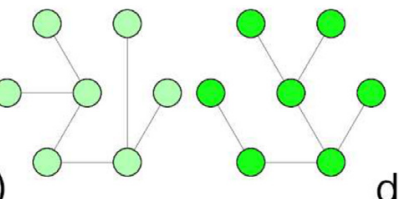

e)

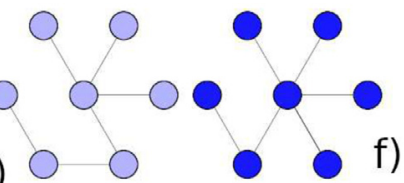

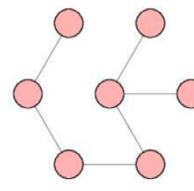

g) b)

d)

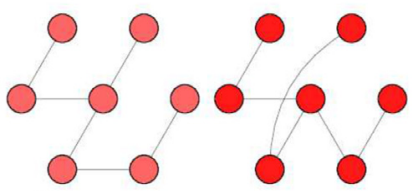

i)
Fig. 4. Graph examples.

"lossy" codes for representing them. For instance, a possible hbsscode could be the vector formed from left to right by the number of vertices, number of edges and its Euler-Poincaré characteristic of the connected graph.

Finally, we are able to define a new tool for topological analysis that informs us about the transference of homological information among boundary scale-space stages.

Definition 4.1. Given a finite cell complex $(K, \partial)$ and an hbssmodel $B S^{2}(K, \partial, t(i))$, the homology boundary scale-space signature $B S^{3}(K, \partial, t(i), m)$ (being $m$ the maximal boundary scale index involved) is a set of hbss-codes, each of one specified by an hbssgraph of the model $B S^{2}(K, \partial, t(i))$.

In the subsequent subsections, we will do some experimentation related to the stability and topological discriminative power of $B S^{2}(K, \partial, b s s)$ and $B S^{3}(K, \partial, n)$, with regards to a particular persistent-based hbss-code. More concretely, each hbss-graph is classified using a persistent hbss-code ( $c r d, b, d, d m s)$, where $c r d$ es is the number of graphs with homological dimension $d m s$ whose boundary-scale "birth" is equal to $b$ and boundary-scale "death" is equal to $d(b, d \leq n)$.
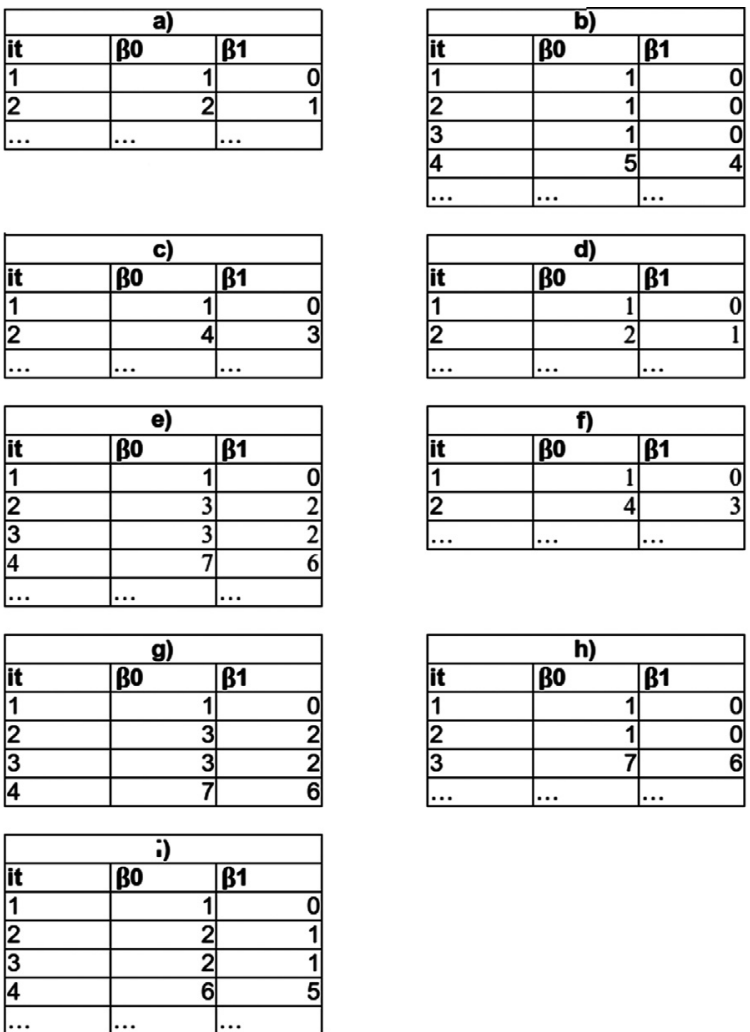

Fig. 5. hbss-Betti sequence corresponding to the graph examples in Fig. 3

\subsection{About stability of the hbss-model}

Fig. 3 a) shows a cell complex of a torus with 832 cells that has been use for experimenting about the stability of the $h b s s$-model. We have tested how the hbss-Betti sequence remains constant after several modifications of the initial complex, including rotation and vertex and cell reordering. The Betti numbers are computed using Algorithm 1. The resulting sequence is shown in Fig. 3 b). Each row corresponds to an iteration in the model. In this example, only the first three iterations are shown, due to the fact that the model stabilizes at this point, and therefore the numbers keep constant in the following iterations.

\subsection{Analysing graphs using hbss-model}

The sequence of Betti numbers of the boundary scale-space chain complexes of the hbss-model given in an increasing order with regards the boundary scale (from now on, hbss-Betti sequence) is a true invariant for isomorphism of geometric cell complexes. This is due to the fact that each chain complex of a certain boundary-scale index can be seen as an appropriate reorganization of the boundary information of the original geometric object with regards to a topological measure (boundary scale) and its homology is a homeomorphic invariant.

We work here exclusively with 1-dimensional geometric complex. Let us start by comparing the hbss-Betti sequence of two homeomorphic graphs.

We also compare the hbss-Betti sequence of various sets of nonisomorphic graphs (see Fig. 4) with the same degree sequence. For orders $n \leq 5$, there are no non-isomorphic trees with the same degree sequence. For $n=6$, the unique cases are shown in Fig. 4 a) and b). The possibilities for $n=7$ are pictured in Fig. 4 from c) to i), being the degree sequences: $(3,3,2,1,1,1,1)$ for c) and d), (4,2,2,1,1,1,1) for e) and f) and (3,2,2,2,1,1,1) for g) and i). 


\begin{tabular}{|c|c|c|c|c|}
\hline & crd & b & d & dim \\
\hline \multirow{2}{*}{ a) } & 1 & 0 & - & 0 \\
\hline & 1 & 1 & - & 1 \\
\hline \multirow{2}{*}{ b) } & 1 & 0 & - & 0 \\
\hline & 4 & 3 & - & 1 \\
\hline \multirow{3}{*}{ c) } & 1 & 0 & - & 0 \\
\hline & 2 & 1 & - & 1 \\
\hline & 4 & 3 & - & 1 \\
\hline \multirow{2}{*}{ d) } & 1 & 0 & - & 0 \\
\hline & 1 & 1 & - & 1 \\
\hline \multirow{3}{*}{ e) } & 1 & 0 & - & 0 \\
\hline & 2 & 1 & - & 1 \\
\hline & 4 & 3 & - & 1 \\
\hline \multirow{2}{*}{ f) } & 1 & 0 & - & 0 \\
\hline & 3 & 1 & - & 1 \\
\hline \multirow{3}{*}{ g) } & 1 & 0 & - & 0 \\
\hline & 2 & 1 & - & 1 \\
\hline & 4 & 1 & - & 1 \\
\hline \multirow{2}{*}{ h) } & 1 & 0 & - & 0 \\
\hline & 6 & 2 & - & 1 \\
\hline \multirow{3}{*}{ i) } & 1 & 0 & - & 0 \\
\hline & 1 & 1 & - & 1 \\
\hline & 4 & 3 & - & 1 \\
\hline
\end{tabular}

Fig. 6. Persistent hbss-code corresponding to the graph examples in Fig. 3.

Fig. 5 shows the corresponding hbss-Betti sequence of such graphs and Fig. 6 shows the persistent hbss-codes. Note that $d$ is not specified, due to the fact that such graphs persist during all the tested iterations (more than 100).

\section{Conclusions}

In this paper, a new paradigm for a scale-space topological analysis of geometric cell complexes is described. The resulting hierarchical graph-based hbss-model is a well-defined and topologically consistent notion, whose nodes represent homology generators of the different hbss stages of the model and whose edges are constructed mirroring a realization of a homology generator at boundary scale $i$ into the $i-1$ stage. The ordered sequence of its homological nodes is an invariant for cell complex isomorphism.

This paper primarily focuses on shaping a mathematically robust theory about a novel computational topological tool and procedures to simplify its still difficult, interpretatively speaking, homological information. Accordingly, the experimentation carried out on small instances fundamentally deals with the understanding, interpretability and stability (under homotopy-preserving changes) of the model and its behavior within the context of geometrical cell complexes of dimension 1 .

Future efforts need to be devoted to solve important problems:

- to analyze hbss-models for boundary operation sequence with transition maps exclusively preserving Euler-Poincaré characteristic.

- to visualize the homological hole information of the hbss-model in terms of delineations and/or cuts composed by set of cells of the original geometric cell complex;

- given an hbss-code, to try to derive an invariant for cell complex isomorphism;

- to design a parallel algorithm for computing the hbss-model based on HSF model.

- for any geometric cell complex, to fix a priori a boundary scale index such that beyond it, we can guarantee that the homological information provided by the hbss-model becomes "stable" and uninformative;
- to describe a balanced hbss-model in terms of both boundary and coboundary scales;

- to develop a bss-model exclusively in terms of homotopy information of abstract cell complexes;

- to show the usefulness of this approach in computer vision and pattern recognition applications. The generation of an efficient and functional computational toolbox to analyze large amounts of data using this new scale-based representation model is a priority for the authors.

\section{Declaration of Competing Interest}

The authors declare that they have no known competing financial interests or personal relationships that could have appeared to influence the work reported in this paper.

\section{Acknowledgments}

This work has been supported by the Spanish research projects (AEI/FEDER,UE) TOP4COG:MTM2016-81030-P, TEC201677785-P, and the VPPI of the University of Seville.

\section{References}

[1] A. Zomorodian, G. Carlsson, Computing persistent homology, Discret. Comput. Geom. 33 (2) (2005) 249-274.

[2] H. Edelsbrunner, J. Harer, Persistent homology-a survey., Contemp. Math. 453 (2008) 257-282.

[3] R. Klette, Cell complexes through time, Commun. Inf. Technol. Res.Tech. Rep. 60 (2000).

[4] T. Lindeberg, Scale-Space Theory in Computer Vision, v. 256, Springer Science \& Business Media, 2013.

[5] K.A. Florack L., The topological structure of scale-space images, J. Math. Imaging Vis. 12 (1) (2000) 65-79.

[6] P.T. Jackway, Morphological scale-space, in: Proceedings., 11th IAPR International Conference on Pattern Recognition. Vol. III. Conference C: Image, Speech and Signal Analysis, IEEE, 1992.

[7] J.H. Bosworth, S.T. Acton, Morphological scale-space in image processing, Digit. Signal Process. 13 (2003) 338-367.

[8] R. González-Diaz, B. Medrano, P. Real, J. Sanchez-Pelaez, Algebraic topological analysis of time-sequence of digital images, Lect. Notes Comput. Sci. 3718 (2005) 208-219.

[9] P. Pilarczyk, P. Real, Computation of cubical homology, (co)homology and (co)homological operations via chain contractions, Adv. Comput. Math. 41 (1) (2015) 253-275.

[10] R. González-Díaz, M.J. Jiménez, B. Medrano, P. Real, Chain homotopies for object topological representations, Discret. Appl. Math. 157 (3) (2009) 490-499.

[11] SAGE, Homology. algebraic topological model module, http://doc.sagemath. org/.

[12] J. Koenderink, The structure of images, Biol. Cybern. 50 (5) (1984) 363-370.

[13] R. Ayala, E. Domínguez, F. A.R., Q. A., Homotopy in digital spaces., Discrete Geometry for Computer Imagery. DGCI 2000, 1953, 2000. Lecture Notes in Computer Science

[14] J. Haarmann, M.P. Murphy, C.S. Peters, P.C. Staecker, Homotopy equivalence in finite digital images., J. Math. Imaging Vis. 53 (3) (2015) 288-302.

[15] T. Kong, R. A., Topological Algorithms for Digital Image Processing, 19, 1996.

[16] L. De Floriani, M.M. Mesmoudi, F. Morando, E. Puppo, Decomposing non-manifold objects in arbitrary dimension, Graph. Models 61 (2003) 2-22.

[17] D. Gunther, J. Reininghaus, H. Wagner, I. Hotz, Efficient computation of 3d morse smale complexes and persistent homology using discrete morse theory, Vis. Comput. 28 (10) (2012) 1684-1699.

[18] V. Kovalevsky, Algorithms in digital geometry based on cellular topology, in: 10th IWCIA, 3322, Springer Berlin Heidelberg, 2004, pp. 366-393.

[19] H.A. Carr, G.H. Weber, C.M. Sewell, J.P. Ahrens, Parallel peak pruning for scalable SMP contour tree computation., IEEE 6th Symposium on Large Data Analysis and Visualization (LDAV) (2016) 75-84.

[20] M. Hilaga, Y. Shinagawa, T. Kohmura, T.L. Kunii, Topology matching for fully automatic similarity estimation of $3 \mathrm{~d}$ shapes, in: Proceedings of the 28th Annual Conference on Computer Graphics and Interactive Techniques, 2001, pp. 203-212.

[21] M. Couprie, G. Bertrand, Asymmetric parallel 3d thinning scheme and algorithms based on isthmuses, Pattern Recogn. Letter 76 (2016) 22-31.

[22] C. Pudney, Distance-ordered homotopic thinning: a skeletonization algorithm for 3d digital images, Comput. Vis. Image Underst. 72 (3) (1998) 404-413.

[23] P.K. Saha, G. Borgefors, G.S. di Baja, A survey on skeletonization algorithms and their applications, Pattern Recogn. Lett. 76 (2016) 3-12.

[24] Y.Y. Boykov, M.P. Jolly, Interactive graph cuts for optimal boundary and region segmentation of objects in nd images, in: Proceedings of Eighth IEEE International Conference on Computer Vision, 1, 2001, pp. 105-112. 
[25] C. Fiorio, A Topologically Consistent Representation for Image Analysis: the Frontiers Topological Graph, 1996, pp. 151-162.

[26] P. Lienhardt, Topological models for boundary representation : a comparison with n-dimensional generalized maps, Comput.-Aided Des. 23 (1) (1991) 59-82.

[27] J. Munkres, Elements of Algebraic Topology, Addison Wesley, 1984.

[28] J. Dumas, B. Saunders, G. Villard, On efficient sparse integer matrix smith normal form computations, J. Symb. Comput. 32 (2001) 71-99.

[29] R. Forman, Morse theory for cell complexes, Adv. In Math. 134 (1998) 90-145

[30] C. Delfinado, H. Edelsbrunner, An incremental algorithm for Betti numbers of simplicial complexes on the 3-sphere, Comput. Aided Geom. Design 12 (1995) $771-784$.

[31] V. Robins, P.J. Wood, A.P. Sheppard, Theory and algorithms for constructing discrete morse complexes from grayscale digital images, in: IEEE Transactions on Pattern Analysis and Machine Intelligence, 33, 2011, pp. 1646-1658.

[32] H. Molina-Abril, P. Real, Homological spanning forest framework for 2d image analysis, Ann. Math. Artif. Intell. 64 (4) (2012) 385-409.
[33] A. Romero, J. Rubio, F. Sergeraert, Effective homology of filtered digital images, Pattern Recognit. Lett. 83 (2016) 23-31.

[34] L. De Floriani, U. Fugacci, F. Iuricich, Homological shape analysis through discrete morse theory, Perspectives Shape Anal. (2016) 187-209.

[35] A. Gonzalez-Lorenzo, A. Bac, J.L. Mari, P. Real, Allowing cycles in discrete morse theory, Topol. Appl. 228 (2017) 1-35.

[36] H. Molina-Abril, P. Real, Homological optimality in discrete morse theory through chain homotopies, Pattern Recogn. Lett. 33 (11) (2012) 1501-1506.

[37] F. Díaz-del Río, P. Real, D. Onchis, A parallel homological spanning forest framework for 2d topological image analysis, Pattern Recogn. Lett. 83 (2016) 49-58.

[38] S. Lefschetz, Algebraic Topology, 27, American Mathematical Society Colloquium Publications, 1942.

[39] S. Eilenberg, S. Mac Lane, 1954, On the groups $h(\pi, n)$, i, ii, iii, Annals of Math 5860 (1953). 55-106,48-139, 513-557

[40] N. Levine, A characterization of pseudo-chain mappings in Mayer complexes, Am. Math. Month. 68 (3) (1961) 259-262. 